\title{
UNDERSTANDING THE CHARACTERISTICS OF L-ASCORBIC ACID-MONTMORILLONITE NANOCOMPOSITE: CHEMICAL STRUCTURE AND BIOTOXICITY
}

\author{
Bor-Yann Chen ${ }^{1}$, Yuan-Haun LeE ${ }^{2}$, Wu-Ching Lin ${ }^{2}$, Feng-Huei Lin ${ }^{2}$, King-Fu Lin ${ }^{2}$ \\ ${ }^{1}$ Department of Chemical and Materials Engineering, National I-Lan University, I-Lan, Taiwan \\ ${ }^{2}$ Department of Material Science and Engineering, National Taiwan University, Taipei, Taiwan
}

\begin{abstract}
Following the previous study to intercalate a 5-fluorouracil (5-FU) into the interlayer spacing of montmorillonite (MMT) for colorectal cancer treatment, we selected a safe and non-pathological microorganism, Saccharomyces cerevisiae, as indicator microorganism to proceed risk assessment on MMT for feasibility study. Dose-response analysis from the perspective in toxicology was conducted to reveal the toxicity rankings of MMT, L-ascorbic acid (LAA) and montmorillonite-Lascorbic acid (MMT-LAA) composites. This study also tended to intercalate LAA into MMT, observing chemical-structure stabilities of LAA-MMT composites. According to infrared spectroscopic analysis via FTIR, the lattice-vibration peaks of the distorted tetrahedron of $\mathrm{SiO}_{4}$ for MMT were ranged in 400-1200 $\mathrm{cm}^{-1}$. X-ray diffraction analysis also addressed the layer spacing changes in the MMT lattice with $\mathrm{pH}$ changes. The variation in the [001] $d$-spacing changed from 12.4 to $19.4 \AA$ with the increased $p H$, allowing higher intercalation of viable compounds (e.g., LAA) for controlled-release applications. Compared to MMT, LAA, the lowest toxicity (e.g., $E C_{50}=3.77^{\circ} 10^{5} \mathrm{ppm}$ ) of the hybrid MMT-LAA also strongly suggested that MMT-LAA composite is safe to be used for further applications. In contrast, the lowest $E C_{0}(0.564 \mathrm{ppm})$ suggested the most sensitive characteristics for MMT-LAA composite to induce the toxic response from no-effect condition. MMT is thus feasible for intercalation of LAA in controlled release to $\mathrm{pH}$ changes; particularly, MMT-LAA is considered non-toxic for further applications.
\end{abstract}

Biomed Eng Appl Basis Comm, 2006(February); 18: 30-36.

Keywords: L-ascorbic acid (LAA), montmorillonite, dose-response analysis, saccharomyces cerevisiae

\section{INTRODUCTION}

The structure of montmorillonite (MMT) is an octahedral laminated sheet, sandwished between

Received: Oct. 11, 2005; Accepted: Jan. 6, 2006

Correspondence: Yuan-Haun Lee, Professor

Department of Material Science and Engineering,

National Taiwan University, Taipei, Taiwan

E-mail: yhlee@ntu.edu.tw tetrahedral silicate layers [1]. Due to the excellent cation exchange property, it could form many nanocomposites with different organic compounds within the interlayer spacing of ca. 0.9 to $1.2 \mathrm{~nm}$ in MMT [2]. In the previous study [3], 5-fluorouracil (5FU) was successfully intercalated into the interlayer spacing of MMT for the feasibility in colorectal cancer treatment. Apparently, the composite of 5FU/montmorillonite achieved a promising goal of 
releasing $5-\mathrm{FU}$ in situ to treat colocrectal cancer in clinical application [3-5]. Prior to indications of MMT as a good carrier for drug design, Lee et al. [6] also examined acute effects of MMT using wistar rats as an in vivo animal model, clearly revealing the harmless nature of MMT. Evidence in biochemical analysis on mice administered with high doses of MMT also indicated that MMT is considered safe for practical use. To provide a quantitative comparison on toxicity of MMT and other related composites, Saccharomyces cerevisiae (or baker's yeast) was also used as an indicator microorganism to confirm that MMT alone was less toxic than others MMT composites via doseresponse analysis. Apparently, MMT alone could be considered nontoxic in characteristics to $S$. cerevisiae and Wistar rat for myriads of applications. Several studies [7-8] also have been presented the sorption of cation on the MMT, and many of these were conducted over appropriate $\mathrm{pH}$ ranges. Owing to these specific characteristics, MMT could thus be very likely feasible to be a candidate drug carrier in medical uses.

L-ascorbic acid (LAA; i.e., vitamin C, a watersoluble vitamin) contains a variety of biological, pharmaceutical and dermatological functions; for example, it can promote collagen biosynthesis, provide photoprotection, scavenge free radical, cause melanin reduction and enhances the immunity (e.g., anti-viral effect) [9-11]. From the perspective in biochemistry, these functions are closely related to the so-called antioxidant properties of this compound. However, LAA is very unstable to exposures in air, light, moisture, heat, metal ions, oxygen, and base, since it is easily decomposed into biologically inactive compounds such as oxalic acid, L-xylonic acid, Lthreonic acid, and L-lyxonic acid [9]. Evidently, applications of vitamin $\mathrm{C}$ in various fields (e.g., dermatology and pharmacology) are limited unless these limitations have been overcome. To improve chemical stability of LAA, several extensive attentions have been paid on encapsulation and immobilization of LAA [11, 12-14] using liposome, microemulsions, and liquid crystals.

Herein, we introduced a new method with practical value of intercalating and stabilizing vitamin $\mathrm{C}$ within an inorganic layered material MMT. Meanwhile, toxicity assessment also pointed out the margin of safety and toxicity rankings for feasibility of LAA-MMT composites in practical applications.

\section{MATERIAL AND METHOD}

\subsection{Montmorillonite Purification}

The crude clay of bentonite in Tai-dong, Taiwan contained several kinds of clay that includes chlorite, kaolinite, illite, and montmorillonite [15-18]. Montmorillonite is about $30-40 \%$ by weight in unearthed bentonite. The purification process is briefly described as follows: One kilogram crude clay was dispersed in $4.0 \mathrm{~L}$ of distilled water and then maintained at room temperature for 3 days to be swelled up by all clay minerals. The clay solution was then poured into a filtration tank and gently stirred at a rate of ca. $60 \mathrm{rpm}$. Particles left on the filtration tank were discarded and all the supernatant was collected. The filtered solution was then centrifuged $(700 \mathrm{x} \mathrm{g})$ twice. The supernatant was discarded and precipitates were dried by freeze-drying at $-40^{\circ} \mathrm{C}$ for 3 days. The dried powder (size about $2.5 \mu \mathrm{m}$ ) was classified as pure montmorillonite for later experimental use.

\subsection{SEM/EDS Analysis}

The surface morphology and element component of montmorillonite was examined by scanning electron microscope (SEM) at $15 \mathrm{kV}$ of accelerating voltage (model number LEO 1530). The samples used for morphology examination were also prepared by compacting the nanocomposite powders into a pellet. The powdered samples placed on a microscope holder were coated with gold in a vacuum (ca. 10-4 torr) chamber.

\subsection{XRD Analysis}

The XRD patterns for the mineralogy of montmorillonite were obtained by a RIGAKU Geigerflex diffractometer equipped with $\mathrm{Cu} \mathrm{K} \alpha$ X-ray radiation, operating at $40 \mathrm{kV}$ and $30 \mathrm{~mA}$. The diffraction patterns were ranged in between angles (2 $\theta$ ) of $1-10^{\circ}$ running at a scanning rate and counting step size of $0.6^{\circ} / \mathrm{min}$ and $0.01^{\circ}$, respectively.

\subsection{Fourier Transform Infrared Spectroscopy}

The Fourier transform infrared (FT-IR) spectra using $\mathrm{KBr}$ pressed disk technique were conducted by Jasco 410 Fourier transform infrared spectrometer. For each sample, ca. $0.90 \mathrm{mg}$ organo-clay and $63 \mathrm{mg} \mathrm{KBr}$ were weighted and then were grounded in an agate mortar for 10 minutes prior to pellet making. The spectra were collected for each measurement over the spectral range of $400-4000 \mathrm{~cm}^{-1}$ with a resolution of 4 $\mathrm{cm}^{-1}$.

\subsection{Dose-response Analysis}

Probit analysis [19] was adopted to reveal dose response curves for toxicity evaluation in response to various montmorillonite solutions. The probit model postulates that the tolerance capacity of individuals with respect to toxic material (e.g., MMT, MMT composites) in a $S$. cerevisiae population is Log- 
normal distribution. A semilogarithmic plot of montmorillonite concentration versus the provoked response is thus assumed to reveal a linear relation. The probit model converts sigmoid-shaped doseresponse curve into a linear normal equivalent deviation (NED) scale. For example, the $50 \%$ and $84.1 \%$ response correspond to unit in the model equals NED scale plus 5 (see Table I in [19]). The conversion formulae can be shown as follows:

$$
\begin{aligned}
& \mathrm{Y}=\mathrm{A}+\mathrm{B} \log \mathrm{Z} \\
& \mathrm{P}=\frac{1}{2}\left\{1+\operatorname{erf}\left(\frac{Y-5}{\sqrt{2}}\right)\right\} \\
& \operatorname{erf}(x)=\frac{2}{\sqrt{\pi}} \int_{0}^{x} e^{-\xi^{2}} d \xi
\end{aligned}
$$

where A and B denote the intercept and slope of dose-response relation, $\mathrm{Z}$ and $\mathrm{Y}$ are montmorillonite concentration (ppm) and probit unit, respectively; $\mathrm{P}$ is the response $(\%)$ corresponding to montmorillonite concentration, $\operatorname{erf}(\mathrm{x})$ is an error function. Note that the response variable is normalized to be located between 0 and 1 . The conversion relation between the probit unit and provoked response is listed elsewhere (Table I in [19])

Table I . The component of montmorilloniu (Two major metal element of montmorillonite are $\mathrm{Si}(34.42 \%)$ and $\mathrm{Al}(22.64 \%))$

\begin{tabular}{|c|c|c|c|}
\hline Element & wt\% & Element & wt $\%$ \\
\hline 0 & $25.26 \%$ & $\mathrm{Si}$ & $34.42 \%$ \\
\hline $\mathrm{Na}$ & $1.44 \%$ & $\mathrm{Ca}$ & $7.52 \%$ \\
\hline $\mathrm{Mg}$ & $1.67 \%$ & $\mathrm{~K}$ & $4.13 \%$ \\
\hline $\mathrm{Al}$ & $22.64 \%$ & $\mathrm{Fe}$ & $2.93 \%$ \\
\hline
\end{tabular}

\section{RESULT AND DISCUSSION}

\subsection{XRD Analysis}

As shown in Fig. 1(a), montmorillonite is one kind of bioinert clay minerals with fine grain and large inter-planar spacing, in particular located in the (001) plane, indicating superior capability to intercalate large molecules into the interlayer space at the (001) plane. It was shown that the [001] diffraction peak of the montmorillonite appears at $2 \theta=7.2^{\circ}$. The characteristic basal spacing $\mathrm{d}$ is $12.4 \AA$ as calculated from Bragg's Law (Eq. (4)) as follows:

$$
n \lambda=2 d \sin \theta,
$$

where $n, \lambda, \theta$, and $\mathrm{d}$ are an integer, the wavelength, the glancing angle of incidence and the interplanar spacing of the clay layers, respectively. For example, plugging $n=1, \lambda=1.54 \mathrm{~nm}, \theta=7.2^{\circ}$ into eq. (4), we might obtain $d=12.4 \AA$. This value is consistent with the reported $d_{001}$ which characterized a single layer of hydrates-12.5 $\AA$ in natural Namontmorillonite [20]. The $d$-spacing difference is as calculated from Eq. (5) as follows:

$$
d_{001}(\text { at } \mathrm{pH} 3,7,10)-d_{001}=\triangle d
$$

For example, plugging $d_{001}$ (at pH 3) $=15.4 \AA$, $d_{001}=12.4 \AA$ into eq. (5), we might obtain $\triangle d=3.0$ $\AA$. As shown in Fig. 1(b), the XRD pattern of MMTLAA composite at $\mathrm{pH} 3$ revealed that the diffraction peak was shifted from $2 \theta=7.2^{\circ}$ (i.e., MMT alone) to $5.8^{\circ}$ and the $d$-spacing increased from $12.4 \AA$ to 15.4 $\AA$, since L-ascorbic acid particles were intercalated within montmorillonite layers. Fig. 1(c), (d) also showed a layer with the basal spacing of $15.5 \AA$ ( $2 \theta$ $\left.=5.7^{\circ}\right)$ and $19.3 \AA\left(2 \theta=4.7^{\circ}\right)$ at $\mathrm{pH} 7$ and 10 , respectively. These results suggested that L-ascorbate anions might be absorbed or intercalated within the inorganic layers, particularly in wider basal $d$-spacing at higher pHs (e.g., from $\mathrm{pH} 7$ to 10). Fig. 2 clearly showed that the $\triangle d$ of the MMT-LAA expanded with the increasing $\mathrm{pH}$ at $3,7,10$. The $d$-spacing increased slightly at $\mathrm{pH}$ increased from 3 to 7 , but significantly increased from 7 to 10 . Due to such a specific characteristics, it is appropriate to be selected as the drug carrier (refer to [3] for an example) at $\mathrm{pH}$ alterations.

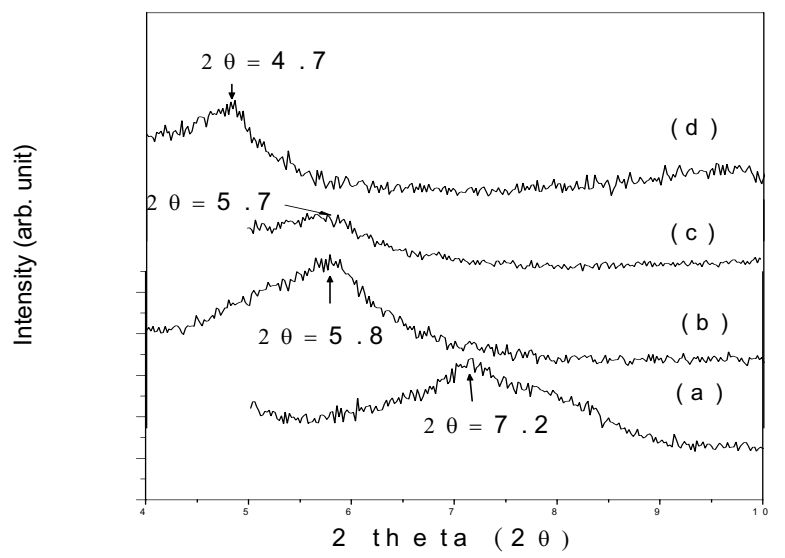

Fig 1(a). X-ray diffraction pattern of montmorillonite (MMT) alone at $\mathrm{pH} 7$; (b) MMTLAA at pH 3; (c) MMT-LAA at pH 7; (d) MMTLAA at pH 10. 


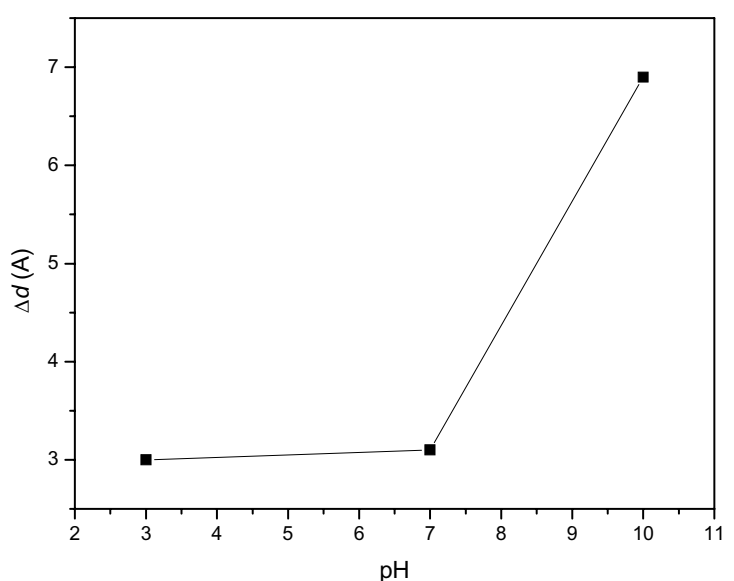

Fig 2. The relationship for $\triangle d$ of MMT versus pH.

\subsection{SEM/EDS Analysis}

After MMT purified from the crude clay of bentonite, it still kept its layer structure and uniformed fine grains to be observed from scanning electron micrograph (SEM) as shown in Fig.3 (a). This photograph showed crumpled platelets that looked like gypsum flower [21]. In addition, as shown in Fig. 3(b)-(d), SEM images of MMT-LAA powders at different $\mathrm{pH}$ value (i.e., $3,7,10$ ) indicated spherical aggregates on the surface of MMT. At $\mathrm{pH} 3, \mathrm{XRD}$ patterns showed that owing to the tightly closed structure in MMT, fewer particles could penetrate into the interlayer. At $\mathrm{pH}$ 10, the layers of MMT opened widely and thus much fewer particles covered on the surface of MMT due to significant intercalation within layers. These also pointed out a marked rise in the capacity for intercalation over $\mathrm{pH}$ changes. As indicated in Table II, the most abundant chemical composition of purified MMT analyzed by EDS are metallic $\mathrm{Si}(34.42 \%)$ and $\mathrm{O}(25.26 \%)$, suggesting $\mathrm{SiO}_{2}$ as a major building block in structure. The chemical formula of the purified MMT could be termed $\left\lceil\left(\mathrm{Al}_{0.61} \mathrm{Fe}^{\mathrm{II}}{ }_{0.04} \mathrm{Mg}_{0.12}\right) \mathrm{Si}_{0.88} \mathrm{O}_{2.68}\right] \mathrm{Na}_{0.1} 0 \mathrm{~K}_{0.05}$.

\subsection{FT-IR Analysis}

The Fourier transform infrared spectra of MMT, MMT-LAA were shown in Fig. 4. As shown the Fig. 4(a) in, the typical bands in spectra of MMT usually appeared to be under $1500 \mathrm{~cm}^{-1}$ due to the absence of H-bond (ca. 3200-3600 $\mathrm{cm}^{-1}$ ) in MMT. Fig. 4(b)-(d) showed the spectra of MMT-LAA composites for various $\mathrm{pHs}$ at $3,7,10$. The bands of 520 and $470 \mathrm{~cm}^{-1}$ (arrow marked at ca. $480 \mathrm{~cm}^{-1}$ ) were assigned to Si-O$\mathrm{Al}$ (octahedral $\mathrm{Al}$ ) and $\mathrm{Si}-\mathrm{O}-\mathrm{Si}$ bending vibrations, respectively. The $\mathrm{OH}$ bending band appears at ca. 915 $\mathrm{cm}^{-1}$ (i.e., Al-OH) [22]. Gradual decreases in the

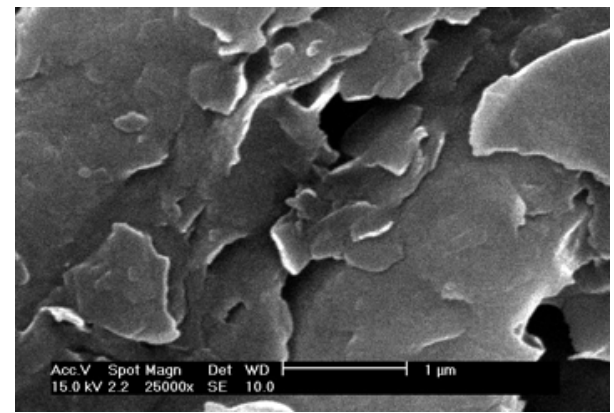

(a)

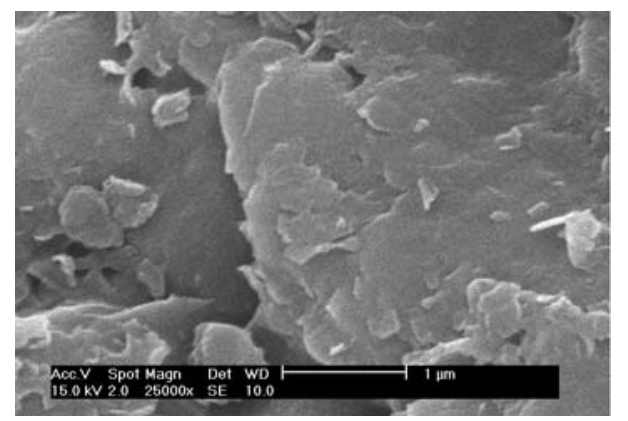

(b)

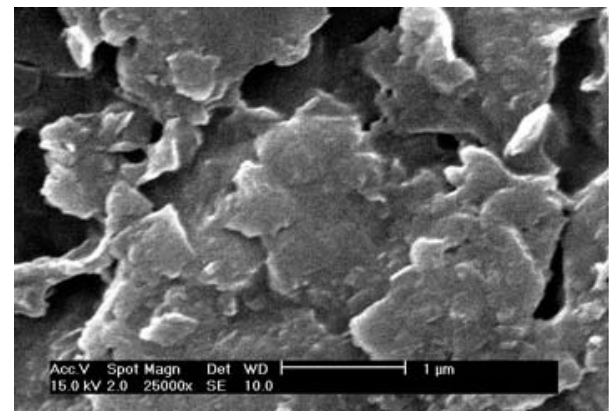

(c)

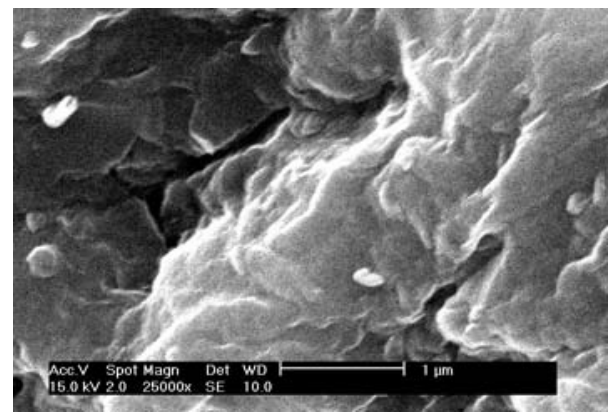

(d)

Fig 3. SEM image of (a) montmorillonite surface $(25,000 X)$.at $\mathbf{~ P H ~ 7 ; ( b ) ~ M M T - L A A ~ s u r f a c e ~}(25,000 X)$ at pH 3; (c) MMT-LAA surface $(25,000 \mathrm{X})$ at $\mathrm{pH} 7$; (d) MMT-LAA surface $(25,000 X)$ at $\mathrm{pH} 10$. 
Table II. Critical effective concentrations of different additives to Saccharomyces cerevisiae predicted from the probit model.

\begin{tabular}{|c|c|c|c|c|c|}
\hline & $\mathbf{E C}_{\mathbf{0}}$ & $\mathbf{E C}_{\mathbf{2 0}}$ & $\mathbf{E C}_{50}$ & $\mathbf{E C}_{100}$ & $\mathbf{Y}=\mathbf{A}+\mathbf{B} \operatorname{logZ}$ \\
\hline $\begin{array}{c}\text { Montmorillonite } \\
\text { (MMT) }\end{array}$ & 34.1 & $4.11 \times 10^{3}$ & $5.41 \times 10^{4}$ & $7.36 \times 10^{8}$ & $\mathrm{Y}=1.45+0.751(\operatorname{logZ})$ \\
\hline $\begin{array}{c}\text { L-Ascorbic acid } \\
(\text { LAA })\end{array}$ & 21.5 & $3.92 \times 10^{3}$ & $6.48 \times 10^{4}$ & $2.02 \times 10^{9}$ & $\mathrm{Y}=1.68+0.690(\log Z)$ \\
\hline MMT-LAA & 0.546 & $3.41 \times 10^{3}$ & $3.77 \times 10^{5}$ & $1.32 \times 10^{13}$ & $\mathrm{Y}=2.71+0.411(\log Z)$ \\
\hline
\end{tabular}

Note: The response variable $\boldsymbol{P}$ is defined as $1-\mu / \mu_{0}$ and $\mathrm{Z}$ denotes the supplemental concentration (ppm).

intensities of $\mathrm{OH}$ bending bands as well as those of the $\mathrm{Si}-\mathrm{O}-\mathrm{Al}$ band with increased $\mathrm{pHs}$ reflected marked decreases in octahedral cations due to deprotonation of LAA to L-ascorbate anion. According to Le Châtelier's principle, an increase in $\mathrm{pH}$ (i.e., hydroxide ion increased) enforced the proton on LAA to be released for neutralization with $\mathrm{OH}^{-}$, causing significantly decrease in intensities of $\mathrm{OH}$ bending band. The broad bands near $1010-1100 \mathrm{~cm}^{-1}$ (e.g. $1016 \mathrm{~cm}^{-1}$ ) were assigned to complex Si-O stretching vibrations in the tetrahedral sheets [23]. The intensity of the $\mathrm{Si}-\mathrm{O}$ band for amorphous $\mathrm{SiO}_{4}$ [22] near 1100 $\mathrm{cm}^{-1}$ increased at lower pHs. The peaks at 1340-1530 $\mathrm{cm}^{-1}$ denoted $\mathrm{C}-\mathrm{H}$ bond appeared and thus caused the adsorbed vibrations. Literature [24-25] indicated that the $\mathrm{C}-\mathrm{H}$ stretching bands most occurred in the 3020$2800 \mathrm{~cm}^{-1}$ region, thus the increase in the bands (ca. $3053 \mathrm{~cm}^{-1}$ ) intensity indicated the more protons and ascorbate ions released to interact with $\mathrm{C}-\mathrm{H}$ bond due to deprotonation of LAA with the increased $\mathrm{pH}$ [22]. Thus, the results might suggest that the L-ascorbate molecules were bounded to the cation in tetrahedron or octahedron, and L-ascorbic acid intercalated into the layers. As shown in Fig. 4(e) for the typical L-ascorbic acid spectrum, the spectra of $\mathrm{C}-\mathrm{H}$ bond stretching vibrations appeared at ca. $2915 \mathrm{~cm}^{-1}$. Peaks at 1275 , $1140 \mathrm{~cm}^{-1}$ denoted the fingerprint regions for the C-2$\mathrm{O}$ stretch vibration mode and the $\mathrm{C}-5-\mathrm{O}$ adsorbed band, respectively [26].

\subsection{Dose-response Analysis}

Comparative differences in toxicity of various MMT composites were determined via dose-response analysis in Fig. 5. According to Ottoboni [26], if effective concentrations ECx (the effective concentration to provoke $x \%$ toxic response; e.g., $\mathrm{EC}_{0}$, $\mathrm{EC}_{50}$ for $x=0,50$ ) for chemical $\mathrm{A}$ are much less than those for chemical B, A is inevitably much more toxic than B. That is to say, a dose-response curve located on the left side clearly indicate high toxicity characteristic of the chemical. The value of $\mathrm{EC}_{0}$ indicated the maximum dose of toxicants showing

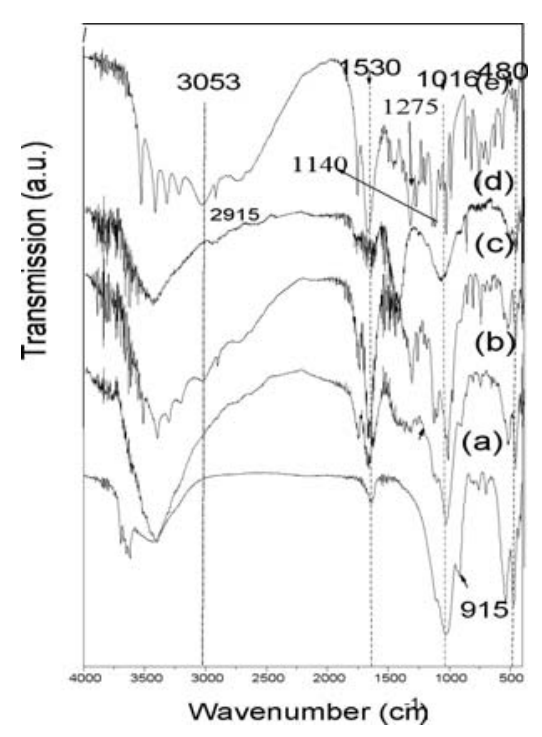

Fig 4. (a) FT-IR spectra of Montmorillonite at pH 7; (b) FT-IR spectra of MMT-LAA at pH 3; (c) FT-IR spectra of MMT-LAA at $\mathbf{p H ~ 7 ; ~ ( d ) ~ F T - I R ~ s p e c t r a ~ o f ~}$ MMT-LAA at pH 10; (e) FT-IR spectra of LAA.

insignificant inhibition to microorganisms. This term may be defined as an indicator variable for revealing "margin of safety" [26]. The toxicity ranking in medium effect dose $\mathrm{EC}_{50}$ (in ppm; shown in Table II) : MMT-LAA $\left(\mathrm{EC}_{50}=3.77 \times 10^{5}\right)>\operatorname{LAA}\left(\mathrm{EC}_{50}=6.48\right.$ $\left.\times 10^{4}\right)>\operatorname{MMT}\left(\mathrm{EC}_{50}=5.41 \times 10^{4}\right)$ clearly suggested that once LAA was added to MMT, this composite was significantly less toxic than both MMT and LAA alone to $S$. cerevisiae. Meanwhile, the ranking of $\mathrm{EC}_{0}$ (in ppm): MMT $\left(\mathrm{EC}_{0}=34.1\right)>$ LAA $\left(\mathrm{EC}_{0}=21.5\right)>$ MMTLAA $\left(\mathrm{EC}_{0}=0.546\right)$ simply suggested that MMT-LAA was more susceptible to induce toxic response from no-effect condition compared to MMT and LAA alone. As shown in the slope factor B of dose-response curve, all curves owned slope factors B less than unity (Table II; Fig. 5; $1.0>0.751$ (MMT) $>0.690$ (LAA) $>0.411$ (MMT-LAA)), suggesting sharper response curves in 


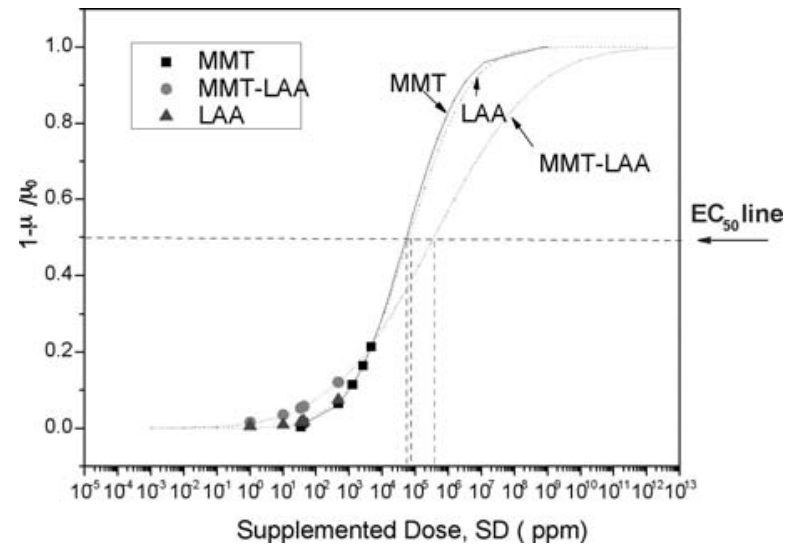

Fig 5. The dose-response curve for Saccharomyces cerevisiae in response to different amended solutions using probit model.

the toxic characteristics for all cases [27-29]. These sharp response curves suggested a broader tolerance range to have toxic response from no-effect concentration $\left(\mathrm{EC}_{0}\right)$ to $100 \%$ response concentration $\left(\mathrm{EC}_{100}\right)$, implying wider administered dose ranges for practical applications. In addition, the smallest value (0.411) of slope B for MMT-LAA directly implied the widest range of tolerance and highest adaptation to our model microorganism, suggesting the most beneficial characteristics of this composite due to significant attenuation in combined toxicity.

It was concluded that using montmorillonite as a composite base for paractical uses (e.g., LAA-MMT) was inevitably biologically-feasible due to the nontoxic characteristics to $S$. cerevisiae. This result was indeed supported that montmorillonite as the "safe" carrier of anticancer drug, 5-FU in previous study [3] was very likely viable. Therefore, this might reveal the significant capability to develop montmorillonite as a newly natural nano-biomaterial for drug release and control in practice.

\section{CONCLUSION}

SEM and XRD results showed that as the $\mathrm{pH}$ increased from 3 to 10 , the interlayer of montmorillonite gradually expanded allowing more Lascorbic acid molecules to penetrate within the interlayer. In addition, results of FT-IR also indicated that a marked rise in ascorbate molecules might be bound to the tetrahedron Si-O bonds, leading to the decreased peaks due to formation of ascorbate anion from LAA molecules at increased pHs (e.g., 3, 7, 10). Dose-response analysis revealed that once MMT was combined with LAA, the $\mathrm{EC}_{50}$ of MMT-LAA was significantly larger than that of MMT and LAA, implying that MMT-LAA was much less toxic than LAA and MMT. In conclusion, MMT might be scientifically feasible to be developed a new form of nanocomposites for further applications in various fields.

\section{REFERENCE}

1. E. G. Ralph, Clay Mineralogy 2nd ed., McGrawHill, New York, 39-41(1968).

2. Y. Laibin, B. Roth Charles, F. Low Philip, "Changes in the Si-O Vibrations of Smectite Layers Accompanying the Sorption of Interlayer Water." Langmuir, 12 (18), 4421 -4429, (1996).

3. F. H. Lin, Y. H. Lee, C. H. Jian, J. M. Wong, M. J. Shieh, C. Y. Wang, "A study of purified montmorillonite intercalated with 5-fluorouracil as drug carrier" Biomaterials 23, 1981-1987(2002).

4. C. Heidelberger, N. K.Chaudhuari, P. Danenberg, "Fluorinated pyrimidines: a new class of tumor inhibitory compounds." Nature 179, 663-666 (1957).

5. N. J. Meropol, P. J. Creaven, N. J. Petrelli, "Metastatic colorectal cancer: advances in biochemical modulation and new drug development" , Seminar Oncology, 22, 509-524 (1995).

6. Y. H. Lee, , T. F. Kuo, B. Y. Chen, Y. K. Feng, Y. R. Wen, W. C. Lin , F. H. Lin, "Toxicity Assessment of Montmorillonite as a Drug Carrier for Pharmaceutical Applications: Yeast and Rats Model." Biomedical Engineering Applications, Basis and Communications, 17(2), 12-18 (2005).

7. Z. Borovec, "Distribution of toxic metals in stream sediments." Chem. Geol., 32, p.45-58 (1981).

8. B. Allard, G.W. Beall, T. Krajewski, J. Radioanal, Nucl. Chem. "Mineral-Contributed Anion Effects on the Retention of Trivalent Actinides in the Environment." 200, p.529 (1995).

9. L. J. Machlin, Handbook of Vitamins, 2nd Ed.; Marcel Dekker, Inc. (1991).

10. A. Bossi, S. A. Piletsky, E. V. Piletska, P. G. Rightti, Turner, A., and Anthony P. F., "An Assay for Ascorbic Acid Based on Polyaniline-Coated Microplates." Anal Chem. 72, 4296(2000).

11. I. Yamamoto, A. Tai, Y. Fujinami, K. Sasaki, S. Okazaki, "Synthesis and characterization of a series of novel monoacylated ascorbic acid derivatives, 6-O-acyl-2-O-alpha-D-glucopyranosylL-ascorbic acids, as skin antioxidants." J. Med. Chem. 45, 462(2002).

12. R. Austria, A. Semenzato, A. Bettero, "Stability of vitamin $\mathrm{C}$ derivatives in solution and topical 
formulations." J. Pharm. Biomed. Anal.15, 795(1997).

13. M. Gallarate, M. E. Carlotti, M. Trotta, S. Bovo, "On the stability of ascorbic acid in emulsified systems for topical and cosmetic use." Int. J. Pharm., 188, 233(1999).

14. A. Semenzato, R. Austria, C. Dall' Aglio, A. Bettero, "HPLC determination of ionic compounds in cosmetic emulsions: Application to magnesium ascorbyl phosphate." J. Chromatogr. A $705,385(1995)$.

15. A. H. Rose, J. S. Harrison, The Yeasts, p. 300, 2nd ed., Academic Press, London (1987).

16. E. W. Charles, The chemistry of clay minerals, Oxford Press, New York (1975).

17. B. B. Zvyagin, Z. G. Pinsker, Structure of Montmorillonite, C. R. Acad. Sci. U.R.S.S., 68, 505-508 (1949).

18. J. Mering, R. Glaeser, "Cations enchangeables dans montmorillonite," C. R. Bull, 5, 61-72 (1953).

19. B.Y. Chen, H.L. Liu, Y.W. Chen, Y.C. Cheng, "Dose-response assessment of metal toxicity upon indigenous Thiobacillus thiooxidans BC1." Process Biochemistry. 39, 735-745 (2004).

20. G. W. Brindley, G. Brown (Eds.), "Crystal Structures of Clay Minerals and their X-Ray Identification." Mineralogical Society, Vol. 5, Chapter 8, London, 197- 248, (1980).

21. R. Marc, "Flourine Route Synthesis of Montmorillonites containing $\mathrm{Mg}$ or $\mathrm{Zn}$ and Characterization by XRD, Thermal analysis, MAS NMR, and EXAFS spectroscopy." Eur. J. Inorg. Chem., Vol. 2001, Issue 11, 2831-2841 (2001).

22. J. Madejova, J. Bujdak, M. Janek, P. Komadel, "Comparative FT-IR study of structure modifications during acid treatment of dioctahedral smectites and hectorite." Spectrochimica Acta Part A, 54, 1397-1406 (1998).

23. R. L. Frost, L. Rintoul, "Lattice vibrations of montmorillonite: an FT Raman and X-ray diffraction study." Applied Clay Science 11, 171183 (1996).

24. J. Madejova, "FTIR techniques in clay mineral studies", Vibrational Spectrosc., 31, 1-10 (2003).

25. M. Kozak, L. Domka, "Adsorption of the quaternary ammonium salts on montmorillonite." Journal of Physics and Chemistry of Solids 65 , 441-445 (2004).

26. B. D. Michael, A. John, A. P. David, "VITAMIN C: Its Chemistry and Biochemistry." Royal Society of Chemistry, Paperbacks, Cambridge 3840, (1991).

27. M. A. Ottoboni, Chapter 8 Chronic toxicity. In: The Dose makes the Poison 2nd Ed. p.79-90, Van
Nostrand Reinhold, ITP, New York (1997).

28. B. Y. Chen, J. S. Chang, "Phenol Degradation and Toxicity Assessment upon Biostimulation to an indigenous Rhizobium Rastonia taiwanesis." Biotechnol, Prog. 21, 1085-1092 (2005).

29. B. Y. Chen, C. H. Wu, J. S. Chang, "An assessment of the toxicity of metals to Pseudomonas aeruginosa PU21 (Rip64)" In press, Bioresour. Technol. (2005). 